\title{
Indications for Proning in Acute Respiratory Distress Syndrome: Expanding the Horizon!
}

\author{
Lokesh K Lalwani $^{1}$, Vinod Sharma ${ }^{2}$, Dhruva Chaudhry ${ }^{3}$, Pawan K Singh ${ }^{4}$
}

\begin{abstract}
Background: Previously prone positioning (PP) was described in addition to invasive mechanical ventilation and it has been known to reduced mortality and improve oxygenation in patients of ARDS. Recently novel timing of prone positioning was described with the use of high-frequency nasal cannula (HFNC) and noninvasive ventilation (NIV) in patients of acute respiratory distress syndrome (ARDS) to avoid the intubation. Here we would like to share a case of severe ARDS where prone positioning was used in a step further ahead.

Case description: A 38-year-old gentleman presented with the complaints of progressive breathlessness, dry cough and fever for 7 days. Patient was diagnosed as a case of H1N1 pneumonia with severe ARDS. Patient was initially managed with invasive mechanical ventilation according to ARDS-Net protocol. Despite persistent hypoxia he was put on prone positioning for consecutive 4 days. Patient was extubated after 10 days of mechanical ventilation and put on HFNC in view of persistent high oxygen requirement. At this point of time, we attempted prone positioning in addition to HFNC. Patient was comfortable on prone position and put himself in the same condition for prolonged periods. His oxygenation showed a remarkable improvement from $\mathrm{PaO}_{2}$ of 63 (before prone positioning) to $136 \mathrm{~mm} \mathrm{Hg}$ (after prone positioning). Oxygen supplementation was later tapered off and subsequently, he improved and was shifted to ward.

Conclusion: Prone positioning is a harmless and still extremely effective intervention which can and should be utilized at all steps of ARDS-management.

Keywords: Acute respiratory distress syndrome, H1N1 influenza, Prone position

Indian Journal of Critical Care Medicine (2020): 10.5005/jp-journals-10071-23480
\end{abstract}

\section{BACKGROUND}

Initially prone positioning (PP) was described in severe acute respiratory distress syndrome (ARDS) patients when it is combined with invasive mechanical ventilation and along with the use of at least 48 hours of neuromuscular blockade. Several meta-analyses have described the efficacy of PP, particularly in the improvement of hypoxemia, ventilation-perfusion mismatch (V/Q ratio), and prevention of ventilator-induced lung injury (VILI). ${ }^{1}$ Recently, a study described the use of PP in combination with high-frequency nasal cannula (HFNC) or noninvasive ventilation (NIV) in moderate-tosevere ARDS patients. It concluded that combining of PP with HFNC or NIV can avoid the need of intubation apart from improvement in oxygen saturation $\left(\mathrm{SpO}_{2}\right)$ and ratio of arterial oxygen to the fraction of inspired oxygen $\left(\mathrm{PaO}_{2} / \mathrm{FiO}_{2}\right){ }^{2}$ Here, we would like to share our own experience of PP with HFNC in a patient of ARDS, albeit at different timing.

\section{Case Description}

A 38-year-old-gentleman presented with complains of acuteonset progressive breathlessness with dry cough and fever for 7 days. He was a never smoker and had no previous known comorbidities. On initial assessment, the patient was tachypneic (respiratory rate: $30 /$ minute) and hypoxic $\left(\mathrm{SpO}_{2}: 76 \%\right.$ at room air which increased to $85 \%$ with oxygen supplementation). His heart rate was 132 per minute. His respiratory system examination revealed bilateral crepitations in mammary, infra-axillary, and infrascapular regions. Clinical evaluation of his other systems (cardiovascular system, central nervous system, and per abdomen) was not showing any significant abnormality. Among blood investigations, complete blood count was largely unremarkable

\begin{abstract}
1,3,4 Department of Pulmonary and Critical Care Medicine, Pandit Bhagwat Dayal Sharma Postgraduate Institute of Medical Sciences, Rohtak, Haryana, India

${ }^{2}$ Department of Pulmonary Medicine, All India Institute of Medical Sciences, Jodhpur, Rajasthan, India

Corresponding Author: Pawan K Singh, Department of Pulmonary and Critical Care Medicine, Pandit Bhagwat Dayal Sharma Postgraduate Institute of Medical Sciences, Rohtak, Haryana, India, Phone: +91 8437013094, e-mail: ga.ps.complete@gmail.com

How to cite this article: Lalwani LK, Sharma V, Chaudhry D, Singh PK. Indications for Proning in Acute Respiratory Distress Syndrome: Expanding the Horizon! Indian J Crit Care Med 2020;24(7):589-591.

Source of support: Nil

Conflict of interest: None
\end{abstract}

(hemoglobin: $13.6 \mathrm{~g} / \mathrm{dL}$, total leukocyte count: $9,200 / \mathrm{mm}^{3}$, platelet count: $3.5 \mathrm{lakh} / \mathrm{mm}^{3}$ ). Similarly, the liver function test (total bilirubin: $1 \mathrm{mg} / \mathrm{DL}$ ), the renal function test (urea and creatinine were 46 and $1 \mathrm{mg} / \mathrm{dL}$, respectively), and the coagulation profile were also within normal limits except for mild transaminitis (alanine transaminase: $65 \mathrm{IU} / \mathrm{L}$, aspartate transaminase: $59 \mathrm{IU} / \mathrm{L}$ ). Chest radiograph was showing bilateral middle- and lower-zone infiltrates (Fig. 1A), and the arterial blood gas ( $A B G)$ analysis was showing hypoxia $\left(\mathrm{PaO}_{2}: 68 \mathrm{~mm} \mathrm{Hg}\right.$ with $\mathrm{FiO}_{2}$ nearly $\left.100 \%\right)$. A provisional diagnosis of severe ARDS was made, given the $\mathrm{PaO}_{2} / \mathrm{FiO}_{2}$ less than 100 , and the patient was intubated. He was ventilated with low tidal volume and high positive end-expiratory pressure (PEEP) strategy as per the ARDS-Net protocol. ${ }^{3,4}$ On evaluation for the etiology of ARDS, his nasopharyngeal swab

(c) The Author(s). 2020 Open Access This article is distributed under the terms of the Creative Commons Attribution 4.0 International License (https://creativecommons. org/licenses/by-nc/4.0/), which permits unrestricted use, distribution, and non-commercial reproduction in any medium, provided you give appropriate credit to the original author(s) and the source, provide a link to the Creative Commons license, and indicate if changes were made. The Creative Commons Public Domain Dedication waiver (http://creativecommons.org/publicdomain/zero/1.0/) applies to the data made available in this article, unless otherwise stated. 

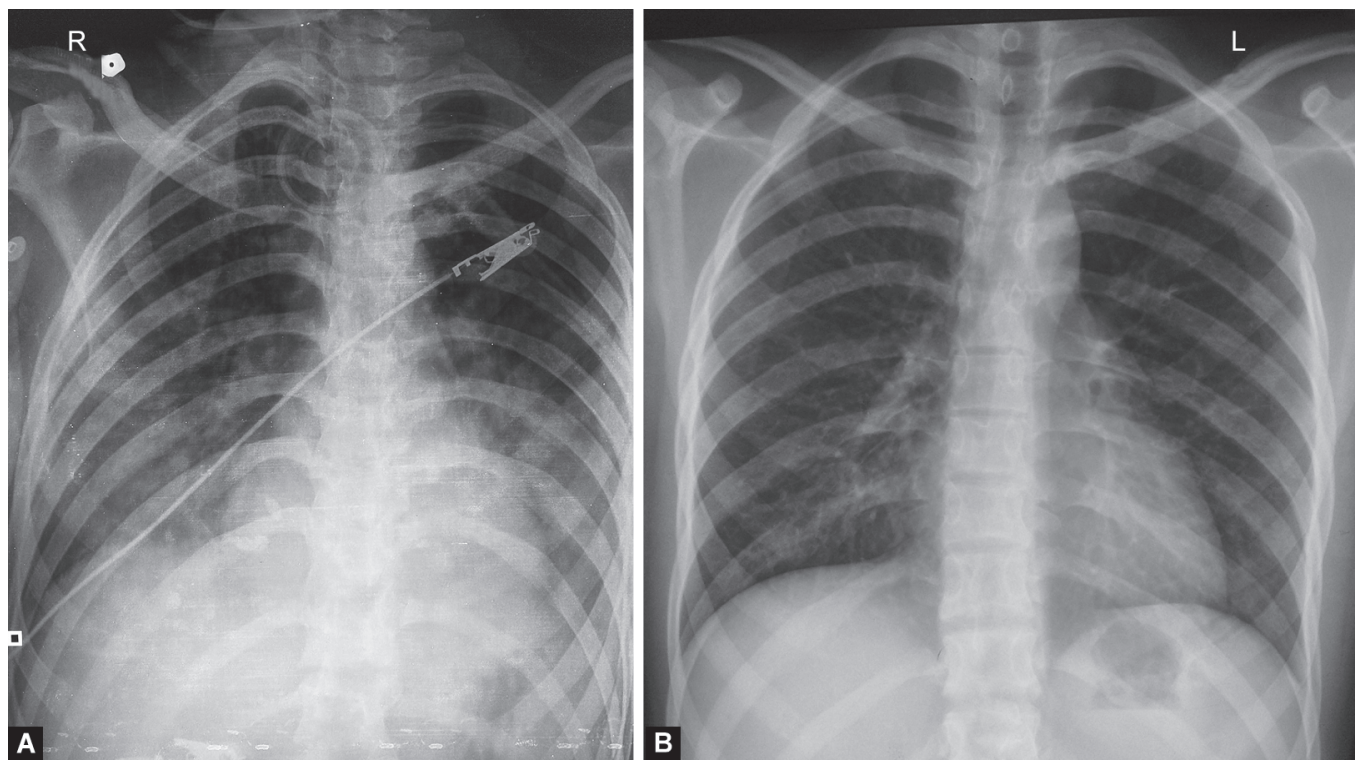

Figs $1 \mathrm{~A}$ and B: (A) Anteroposterior chest radiograph showing bilateral middle- and lower-zone opacities; (B) Posteroanterior chest radiograph showing significantly improved chest condition in comparison with previous imaging

Table 1: Arterial blood gas analysis report of index patient before and after prone positioning on mechanical ventilation and also showing ABG at time of before and after prone positioning on HFNC demonstrating significant improvement in $\mathrm{PaO}_{2}$ values

\begin{tabular}{lcccc}
\hline ABG parameter & $\begin{array}{l}\text { Intubated (before } \\
\text { proning) }\end{array}$ & $\begin{array}{l}\text { Intubated (after } \\
\text { proning) }\end{array}$ & $\begin{array}{l}\text { After extubation/before } \\
\text { prone positioning on HFNC }\end{array}$ & $\begin{array}{l}\text { After prone positioning on } \\
\text { HFNC }\end{array}$ \\
\hline $\mathrm{pH}$ & 7.32 & 7.45 & 7.56 & 7.51 \\
$\mathrm{PaCO}_{2}(\mathrm{~mm} \mathrm{Hg})$ & 45.4 & 38.6 & 32.4 & 48.4 \\
$\mathrm{PaO}_{2}(\mathrm{~mm} \mathrm{Hg})$ & 61 & 107.4 & 64.3 & 136.4 \\
$\mathrm{HCO}_{3}(\mathrm{mEq} / \mathrm{L})$ (bicarbonate) & 26.7 & 32.4 & 34.2 & 38.2 \\
$\mathrm{SpO}_{2}(\%)$ & 88.6 & 93.5 & 91.2 & 98.9 \\
$\mathrm{FiO}_{2}(\%)$ & 100 & 100 & 45 & 45 \\
\hline
\end{tabular}

was sent for $\mathrm{H}_{1} \mathrm{~N}_{1}$ testing (reverse transcription polymerase chain reaction), which came out to be positive and he was started on oseltamivir (75 mg twice daily for 5 days) apart from ceftriaxone and azithromycin (empiric institutional protocol for ARDS). The patient was persistently hypoxic despite high PEEP, recruitment maneuvers, and $100 \% \mathrm{FiO}_{2}$ (Table 1). Subsequently, he was put on the prone position for 16 hours/day for 4 consecutive days. The patient improved clinically, and 10 days later, he was weaned off from mechanical ventilation.

Despite ameliorated chest condition and extubation (Fig. 1B), the patient continued to be tachypneic and hypoxic. During invasive mechanical ventilation, various endotracheal aspirates to look for ventilator-associated pneumonia were done, which all came out to be sterile. He was initiated on HFNC with flow of $45 \mathrm{~L} /$ minute at a $\mathrm{FiO}_{2}$ of $45 \%$. On $\mathrm{HFNC}$, he was comfortable and maintaining a $\mathrm{SpO}_{2}$ of $91 \%$. $\mathrm{His} \mathrm{PaO}_{2} / \mathrm{FiO}_{2}$ ratio was 143 on $\mathrm{ABG}$. At this point of time, the study by Lin Ding et al. ${ }^{2}$ was discussed in our departmental journal club, and it was planned to attempt PP in our index case while being on HFNC. As he was quite cooperative, he was asked to lie in a prone position if he felt comfortable (Fig. 2). He tolerated prone position well, and 1 hour later of PP when ABG was repeated, his $\mathrm{SpO}_{2}$ had increased to $98 \%$ and $\mathrm{PaO}_{2}$ was $136 \mathrm{~mm} \mathrm{Hg}$ on same settings $\left(\mathrm{PaO}_{2} / \mathrm{FiO}_{2}: 302\right)$ (Table 1$)$. In the following course of his ICU stay, he himself was inclined to stay in the prone position for prolonged periods. Over the next 48 hours, his $\mathrm{FiO}_{2}$ was tapered

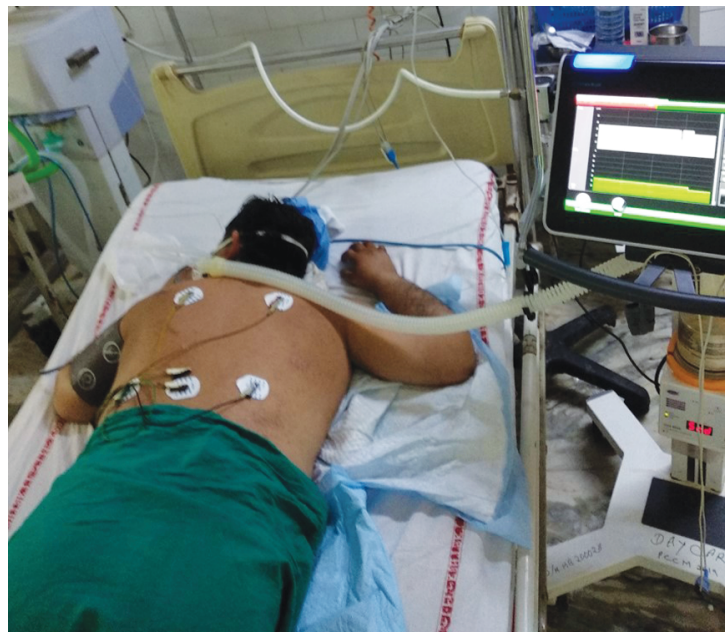

Fig. 2: Image of the patient in prone position on HFNC. Unlike proning on invasive ventilation, on HFNC, patient positioned himself prone and was able to frequently change his neck and limb positions himself

off, and he was shifted to the ward. After a stay of 2 days in ward, he was off oxygen supplementation and mobilizing. All parenteral medicines were stopped and he was discharged on 5th day of ward stay. 


\section{Discussion}

Acute respiratory distress syndrome is one of the most essential indications of mechanical ventilation in ICU. It carries a high mortality rate. In recent past, there have been significant changes in the ventilatory strategies of ARDS apart from low tidal volume ventilations, like proning. ${ }^{5}$ Prone positioning in ARDS first came into limelight after the PROSEVA study where a significant mortality benefit was achieved by proning. Its proposed mechanisms include improvement in lung homogeneity and ventilation/perfusion mismatch. ${ }^{6}$ It is one of the few strategies in ARDS, which have proven mortality benefit when used with invasive mechanical ventilation.

Recently, Lin Ding et al. proposed a novel timing of PP combined with HFNC or NIV in moderate-to-severe ARDS. This trial demonstrated the efficacy of PP when applied early in combination with HFNC, especially in patients with moderate ARDS. The PP was well tolerated, and both HFNC and NIV were superior when combined with PP, especially in preventing intubations. Severe ARDS patients were concluded to be unsuitable candidates for HFNC/NIV+PP. ${ }^{2}$ This article prompted us to use this strategy in one of our patients, albeit at a different timing. The use of post-extubation PP in this particular patient emphasizes on the efficacy of proning at all phases of ventilation. In addition to the study by Ding et al. and our experience with this patient, we feel that PP is an underutilized silver bullet. New modifications in the use of PP are now coming up, like prolonged proning, early proning, and post-extubation proning. ${ }^{7}$ Though there are few contraindications of proning but we strongly feel that these are just relative contraindications and more of technical obstacles instead of absolute ones. Given the high number of ARDS cases in previous pandemics and current COVID-19 pandemic, where the pharmacological panacea is yet to be known, prone can be a valuable tool in the armamentarium. And optimum utilizing of such tools should be explored further without being restricted to the traditional indications only.

\section{Conclusion}

Early use of PP with HFNC or NIV in moderate to severe patients of ARDS, even in subjects who are not on mechanical ventilation, can benefit in avoiding intubations. Our experience in managing the index case with PP and HFNC in the post-extubation phase indicates that PP is a highly effective intervention and can be used at all steps of ARDS management.

\section{Clinical Significance}

Emphasizes on the all-positive aspects of PP in the management of ARDS irrespective of timing. The prone position remains underutilized, and its benefits are still underrecognized.

\section{References}

1. Munshi L, Del Sorbo L, Adhikari NKJ, Hodgson CL, Wunsch H, Meade $\mathrm{MO}$, et al. Prone position for acute respiratory distress syndrome. A systematic review and meta-analysis. Ann Am Thorac Soc 2017;14(Suppl 4):S280-s288. DOI: 10.1513/AnnalsATS.201704-343OT.

2. Ding L, Wang L, Ma W, He H. Efficacy and safety of early prone positioning combined with HFNC or NIV in moderate to severe ARDS: a multi-center prospective cohort study. Crit Care 2020;24(1):28. DOI: 10.1186/s13054-020-2738-5.

3. Griffiths MJD, McAuley DF, Perkins GD, Barrett N, Blackwood B, Boyle $A$, et al. Guidelines on the management of acute respiratory distress syndrome. BMJ Open Respir Res 2019;6(1):e000420. DOI: 10.1136/ bmjresp-2019-000420.

4. Papazian L, Aubron C, Brochard L, Chiche JD, Combes A, Dreyfuss D, et al. Formal guidelines: management of acute respiratory distress syndrome. Ann Intensive Care 2019;9(1):69. DOI: 10.1186/s13613-019-0540-9.

5. Bos LD, Martin-Loeches I, Schultz MJ. ARDS: challenges in patient care and frontiers in research. Eur Respir Rev 2018;27(147). DOI: 10.1183/16000617.0107-2017.

6. Guerin C, Reignier J, Richard JC, Beuret P, Gacouin A, Boulain T, et al. Prone positioning in severe acute respiratory distress syndrome. $N$ Engl J Med 2013;368(23):2159-2168. DOI: 10.1056/NEJMoa1214103.

7. Scholten EL, Beitler JR, Prisk GK, Malhotra A. Treatment of ARDS with prone positioning. Chest 2017;151(1):215-224. DOI: 10.1016/ j.chest.2016.06.032. 